\title{
Analysis of short- and long-range interactions within potential binding sites notably extends the fraction of verified peaks in ChIP-seq data
}

\author{
Anton Tsukanov \\ ICG SB RAS, Novosibirsk, Russia \\ tsukanov@bionet.nsc.ru
}

\author{
Victor Levitsky \\ ICG SB RAS, Novosibirsk, Russia \\ levitsky@bionet.nsc.ru
}

\author{
Tatyana Merkulova \\ ICG SB RAS, Novosibirsk, Russia \\ merkulova@bionet.nsc.ru
}

\begin{abstract}
We developed pipeline for integrative application of various de novo motif search tools to massive sequencing data. While traditional position weight matrices (PWMs) neglect dependencies between positions of motifs, the 'short-range interactions' markov models BAMM/InMode permit only local dependencies, the 'long-range interaction' model SiteGA allows dependencies between arbitrary positions. The massive analysis of ChIP-seq data have shown that the models BAMM/InMode and PWMs recognized similar and significantly overlapped peaks; but the notable fractions of peaks predicted by SiteGA model were not predicted by other models.
\end{abstract}

Keywords - chromatin immunoprecipitation, transcription factor binding site recognition, short- and long-range dependencies between nucleotide positions in binding sites

\section{Motivation and aim}

Regulation of eukaryotic gene expression achieved through compound interaction of various transcription factors (TFs). Development and massive application of next generation sequencing technologies for mapping of $\mathrm{TF}$ binding sites (BS) in genome, in particular ChIP-seq, provides an opportunity to study gene expression regulation in detail. Widely applied approach of TFBS prediction, the position weight matrix (PWM) relied on a relatively short motifs and proposed the additivity of various positions within potential TFBS [1,2]. Recently, to supplement the verification of potential BSs in ChIP-seq data with PWMs, various approaches that taking into account intra-motifs dependencies were applied for wide-genome application, e.g. BaMM [3] and InMode [4]. These approaches use the markov models (MMs), which neglect the additivity assumption through the concept of the order of markov chain, i.e. a short distance for a given position that may contain other dependent nucleotides. Typically, the order of MM is changed from one to five, that's way MMs may be referred as to as 'short-range interaction' models.

In this study, we proposed new 'long-range interaction' de novo motif search model SiteGA that takes into account dependencies between arbitrary positions of sites. SiteGA approach combined discriminant analysis and genetic algorithm to construct a site model [5]. To compare our approach with traditional PWMs and BAMM/InMode models we developed the integrated pipeline for ChIP-seq data verification with multiple de novo motif search models. The aim of our research does not consist in a performance comparison between various complementary motifs recognition tools, rather, we tested whether alternative tools actually capable predicted structurally different motifs.

\section{Methods}

Earlier, the training of SiteGA model for the aligned sequence dataset defined an individual as a set of $\mathrm{N}$ locally positioned dinucleotides (LPD) frequencies [5]. SiteGA optimized the LPD set with genetic algorithm (GA), i.e. the final LPD set is the top-scoring result of the evolution of the population of individuals. The original SiteGA approach [5] required the same length $\mathrm{W}$ of all sequences from the training set; each LPD was defined by location [s, e] within the whole site $[1, \mathrm{~W}]$ and dinucleotide type $\mathrm{d}, 1 \leq \mathrm{d} \leq 16$, for each LPD. For each $\mathrm{LPD} 1 \leq \mathrm{s}$, e $<\mathrm{W}$ and $0 \leq \mathrm{s}-\mathrm{e} \leq 5$. In the de novo SiteGA version we preserved the length of model $\mathrm{W}$ and the fitness function for an individual [5]. Since any de novo motif search works with peaks of various lengths $\{\mathrm{Ln}\}$ (W $\leq \mathrm{Ln}, 1$ $\leq \mathrm{n} \leq \mathrm{N}, \mathrm{N}$ is the number of peaks), we added to the definition of individual the leftmost position and orientation on of the site $\mathrm{W}$ in a peak. Besides earlier applied mutation and recombination operators that changed location or dinucleotide type of each LPD within the site [1, W], de novo model added in GA implementation new mutation and recombination operators. New mutations can move a position or orientation of the window $\mathrm{W}$ in a peak. New recombination allows an exchange between two individuals that have sites in n-th and $\mathrm{m}$-th peaks in certain positions/orientation. For de novo SiteGA models we chose the same site length $\mathrm{W}=30 \mathrm{nt}$ according to previous analysis [5].

We applied the standard cross-validation method to estimate the accuracy of the models. As a result ROC curve represents the dependence between True Positive and False Positive rates (TPR and FPR, respectively). The thresholds for all models was chosen uniformly with the fixed FPR value. As described earlier [6], FPRs were estimated as probabilities of hits for the respective human/mouse whole-genome dataset of upstream regions of human/mouse protein coding genes of length $2 \mathrm{~kb}$. Peaks were classified according to presence/absence of predicted hits of various models. All supported methods (estimation of model accuracy, determination of the model threshold, classification of peaks) were implemented in the python programming language.

\section{Results}

The new pipeline was developed based on the PWM (ChIPMunk, [7]), InMoDe, BaMM and SiteGA models combined with supported methods to search TFBS in ChIPseq data. The pipeline includes several main steps: 1) training models; 2) estimating models accuracy; 3 ) calculating models thresholds; 4) recognition of potential sites in peaks; 5) classification of peaks according the presence of sites and their overlap within a peak sequence. 
Totally, we took over 100 ChIP-seq datasets from the Cistrome database (http://cistrome.org/, [8]) in analysis. We revealed that each model among all tested (PWM, InMoDe, BaMM, SiteGA) able to verify specific peaks that do not verified by any other models. The typical results of pipeline application for NR3C1 ChIP-seq dataset (GEO: GSM1122535, CistromeDB: 37598, cell type: epithelium) are shown in Fig. 1.

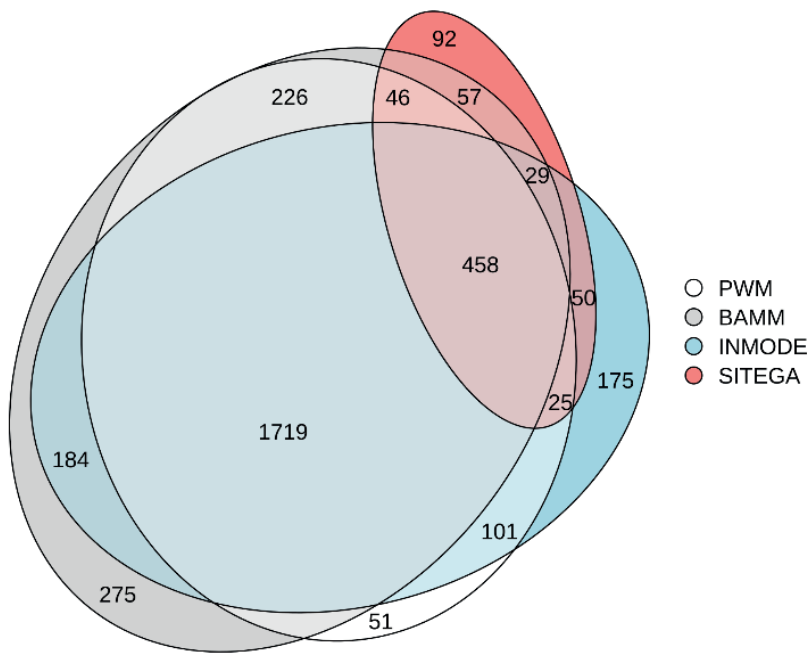

Fig. 1. The Venn diagram of the number of peaks containing predicted sites of sole models, as well as those containing predicted sites of various combinations of two, three and all four models. The diagram is drawn with eulerr [9]. ChIPseq dataset for NR3C1 we extracted from [8]. Totally, the ChIP-seq dataset consisted of 4000 peaks

\section{ACKNOWLEDGMENT}

The work was supported by Russian Foundation for Basic Research (project \# 18-29-13040) and State Budget Project 0259-2019-0008-C-01.

\section{REFERENCES}

[1] Benos P.V. et al. (2002) Additivity in protein-DNA interactions: how good an approximation is it? Nucleic Acids Res., 30(20):4442-4451.

[2] Srivastava D and Mahony S. (in press) Sequence and chromatin determinants of transcription factor binding and the establishment of cell type-specific binding patterns. Biochim Biophys Acta Gene Regul Mech., 194443. doi: 10.1016/j.bbagrm.2019.194443.

[3] Siebert M. and Söding J. (2016) Bayesian Markov models consistently outperform PWMs at predicting motifs in nucleotide sequences. Nucleic Acids Res., 44(13):6055-6069.

[4] Eggeling R. et al. (2017) InMoDe: tools for learning and visualizing intra-motif dependencies of DNA binding sites. Bioinformatics, 33(4):580-582.

[5] Levitsky V.G. et al. (2007) Effective transcription factor binding site prediction using a combination of optimization, a genetic algorithm and discriminant analysis to capture distant interactions. BMC Bioinformatics, 8: 481.

[6] Levitsky V.G. et al. (2019) A single ChIP-seq dataset is sufficient for comprehensive analysis of motifs co-occurrence with MCOT package. Nucleic Acids Res, 47(21): e139.

[7] Kulakovskiy I.V. et al. (2010) Deep and wide digging for binding motifs in ChIP-Seq data. Bioinformatics. 26(20):2622-2623.

[8] Mei S. et al. (2017) Cistrome Data Browser: a data portal for ChIP-Seq and chromatin accessibility data in human and mouse, Nucleic Acids Res., 45(D1):, D658-D662,.

[9] Larsson J. (2019). eulerr: Area-Proportional Euler and Venn Diagrams with Ellipses. $\mathrm{R}$ package version 6.0.0, https://cran.rproject.org/package $=$ eulerr. 